\title{
Peri-Implant Health and the Knowing-Doing Gap-A Digital Survey on Procedures and Therapies
}

\section{OPEN ACCESS}

Edited by:

Kristina Bert1,

Malmö University, Sweden

Reviewed by:

Christian Wehner,

University Dental Clinic Vienna, Austria

Alex Solderer,

University of Zurich, Switzerland

*Correspondence:

Ståle Petter Lyngstadaas spl@odont.uio.no

Specialty section

This article was submitted to

Periodontics,

a section of the journal

Frontiers in Dental Medicine

Received: 17 June 2021 Accepted: 24 September 2021 Published: 25 October 2021

Citation:

Hussain B, Haugen HJ, Aass AM, Sanz M, Antonoglou GN, Bouchard P,

Bozic D, Eickholz P, Jepsen K, Jepsen S, Karaca EO, Kuru BE, Nemcovsky CE, Papapanou PN, Pilloni A, Renvert S, Roccuzzo M,

Sanz-Esporrin J, Spahr A

Stavropoulos A, Verket A, Vražić $D$ and Lyngstadaas SP (2021)

Peri-Implant Health and the Knowing-Doing Gap-A Digital Survey on Procedures and Therapies.

Front. Dent. Med. 2:726607. doi: 10.3389/fdmed.2021.726607

\section{Badra Hussain ${ }^{1}$, Håvard Jostein Haugen ${ }^{1}$, Anne Merete Aass ${ }^{2}$, Mariano Sanz ${ }^{2,3}$, Georgios N. Antonoglou ${ }^{3}$, Philippe Bouchard ${ }^{4,5,6}$, Darko Bozic ${ }^{7}$, Peter Eickholz ${ }^{8}$, Karin Jepsen ${ }^{9}$, Sören Jepsen ${ }^{9}$, Ebru Ozkan Karaca ${ }^{10}$, Bahar Eren Kuru ${ }^{10}$, Carlos E. Nemcovsky ${ }^{11}$, Panos N. Papapanou ${ }^{12}$, Andrea Pilloni ${ }^{13}$, Stefan Renvert ${ }^{14,15,16,17}$, Mario Roccuzzo ${ }^{18}$, Javier Sanz-Esporrin ${ }^{3}$, Axel Spahr ${ }^{19}$, Andreas Stavropoulos ${ }^{20,21}$, Anders Verket ${ }^{2}$, Domagoj Vražić ${ }^{7}$ and Ståle Petter Lyngstadaas ${ }^{1 *}$}

${ }^{1}$ Department of Biomaterials, Institute of Clinical Dentistry, University of Oslo, Oslo, Norway, ${ }^{2}$ Department of Periodontology, Institute of Clinical Dentistry, University of Oslo, Oslo, Norway, ${ }^{3}$ Etiology and Therapy of Periodontal and Peri-Implant Diseases (ETEP) Research Group, Faculty of Odontology, University Complutense of Madrid, Madrid, Spain, ${ }^{4}$ Department of Periodontology, Service of Odontology, Rothschild Hospital, AP-HP, Paris, France, ${ }^{5}$ Université de Paris Garancière, U.F.R. of Odontology, Paris, France, ${ }^{6}$ Université de Paris, URP2496, Orofacial Pathologies, Imaging and Biotherapies Laboratory, Paris, France, ${ }^{7}$ Department of Periodontology, School of Dental Medicine, University of Zagreb, Zagreb, Croatia, ${ }^{8}$ Department of Periodontology, Center of Dentistry and Oral Medicine (Carolinum), Johann Wolfgang Goethe-University Frankfurt, Frankfurt, Germany, ${ }^{9}$ Department of Periodontology, Operative and Preventive Dentistry, Center for Dental, Oral and Maxillofacial Medicine, University Hospital Bonn, Bonn, Germany, ${ }^{10}$ Department of Periodontology, Faculty of Dentistry, Yeditepe University, Istanbul, Turkey, ${ }^{11}$ Department of Periodontology and Implant Dentistry, School of Dental Medicine, Tel-Aviv University, Tel Aviv, Israel, ${ }^{12}$ Section of Oral, Diagnostic and Rehabilitation Sciences, Division of Periodontics, Columbia University College of Dental Medicine, New York, NY, United States, ${ }^{13}$ Section of Periodontology, Department of Dental and Maxillofacial Surgery, Sapienza University of Rome, Rome, Italy, ${ }^{14}$ Department of Health Sciences, Kristianstad University, Kristianstad, Sweden, ${ }^{15}$ Department of Health, Blekinge Institute of Technology, Karlskrona, Sweden, ${ }^{16}$ School of Dental Science, Trinity College, Dublin, Ireland, ${ }^{17}$ Faculty of Dentistry, The University of Hong Kong, Hong Kong, SAR China, ${ }^{18}$ Division of Maxillo-Facial Surgery, University of Turin, Turin, Italy, ${ }^{19}$ Dental School, Faculty of Medicine and Health, The University of Sydney, Sydney, NSW, Australia, ${ }^{20}$ Division of Regenerative Dental Medicine and Periodontology, University Clinics of Dental Medicine CUMD, University of Geneva, Geneva, Switzerland, ${ }^{21}$ Department of Periodontology, Faculty of Odontology, Malmö University, Malmö, Sweden

Objectives: Peri-implant tissue maintenance and treatment is becoming a serious challenge in implantology. With increasing numbers of implants being placed, more cases of peri-implant mucositis and peri-implantitis is seen. A digital survey on peri-implant disease management was issued to experts in periodontology and implantology to identify the tools and procedures most commonly used today to treat peri-implant diseases and successfully manage peri-implant health. The primary aim was to assess whether there is consensus in the choice of treatment to manage peri-implant diseases and to prevent their recurrence once treated. The secondary aim was to obtain insight into future protocols and /or devices, and the research and development needed.

Materials and Methods: Participants in this digital survey were professionals specialising in periodontology, oral surgery, and implant dentistry. The questionnaire included both a series of closed- and open-ended questions. A total of 16 countries participated. The survey was sent by e-mail to 70 individuals, 66 received the survey and 37 of receivers responded, two of the participants were excluded due to insufficient filling of the survey. In the end 35 respondents completed the survey. 
Results: Respondents agree that the efficacy of mechanical and chemical decontamination of implant surfaces needs to be improved and better documented. It is a common opinion that the current remedies, mostly adapted from periodontal practises, do not provide effective and reliable clinical outcomes when treating peri-implant ailments. There is a general agreement amongst experts that regularly scheduled (3-6-month intervals) maintenance treatments are essential for maintaining peri-implant health in patients experiencing implant complications. Respondents are also concerned about unnecessary use of systemic antibiotics for managing peri-implant health.

Conclusion: Regardless of agreements in parts, there was no observed consensus on the most effective treatment options for treating peri-implantitis. The experts all agree it is an urgent need for well-designed, long-term follow-up randomised and controlled clinical trials comparing interventions to provide an evidence-based strategy for peri-implant health management.

Keywords: digital survey, peri-implantitis, implantology, peri-implant health management, implant surface cleaning, antibiotic resistance

\section{INTRODUCTION}

Peri-implant diseases and complications have until recently attracted little attention from either industry or clinicians. However, peri-implant complications were already described in 1965 when Levignac discussed the clinical observations associated with peri-implant inflammation and bone loss and labelled the condition "peri-implantitis" (1). Early on, periimplantitis was categorised as a chronic, infectious pathological condition of the peri-implant tissues, including bone (2) and further defined as an infectious disease with a high similarity to chronic periodontitis $(3,4)$.

The typical signs and symptoms of peri-implant mucositis and peri-implantitis have been comprehensively discussed in several journals and consensus conferences (5-12). The agreed upon definition of peri-implant mucositis is an inflammatory lesion that resides in the mucosa surrounding the implant, while periimplantitis is broadly defined as peri-implant inflammation that also affects the supporting bone $(6,11)$. It is essential to recognise peri-implant health to distinguish it from disease and establish good clinical routines to prevent implant-related diseases and their progression, as well as timely and appropriate interventions when needed $(8,13)$.

Today, most clinicians and clinical researchers recognise that implant-associated mucositis and loss of peri-implant bone are progressive conditions that demand professional intervention $(14,15)$. A prerequisite in intervention against peri-implant diseases and in restoring peri-implant health is control of the local infection and inflammation. Restoring peri-implant health involves clinical procedures for the reduction/elimination of the subgingival biofilm on implant surfaces or implant-related components. Removal of the biofilm is performed by careful cleaning and decontamination of implant surfaces and the affected tissues (16).

Evidently, with an increasing number of dental implants in function and longer follow-up periods, peri-implant disease cases are also on the rise (17). Peri-implant disease treatment and maintenance of healthy peri-implant tissues have become a focus in implant dentistry. When evaluating the evidence for the efficacy of multiple treatment alternatives and procedures, several non-surgical implant surface cleaning protocols and surgical interventions are proposed. Such interventions range from access flap surgery to resective or regenerative approaches or a combination of thereof with the combination of cleaning and detoxification of the implant surface modifications and the use of bone grafts and biologicals. In general, the evidence for these interventions' efficacy is conflicting $(18,19)$, especially for the non-surgical approaches, treatment outcome is unpredictable (18). Most of these modalities of treatment are adaptations from procedures aimed at treating periodontitis. However, a few devices and techniques have been especially researched and developed for implants and peri-implant diseases (20).

Established implant surface decontamination protocols include access by open flap surgery, micro-surgery, or noninvasive/non-surgical procedures combined with mechanical debridement and cleaning of the exposed surfaces, mainly using curettes, brushes, ultrasonic devices, air-abrasives, or lasers (21-25). Implantoplasty, the modification of the implant surface that includes thread removal and smoothening of the implant surface using rotating instruments, is also used by some clinicians, often in combination with other cleaning procedures (26-30). There are varying reported results and many opinions regarding the different procedures (20). Surgical intervention is recommended when the operator cannot obtain proper access to the infected implant surface $(19,20,31)$. Finally, when it comes to implant debridement and surface decontamination, so far no specific decontamination protocol has been shown to be superior to others, whether in surgical or non-surgical treatments (31).

Studies have shown that adjunctive use of both systemic and local antibiotics or antiseptics can temporarily reduce bleeding on probing when treating peri-implant mucositis and peri-implantitis (31). However, there is no generally accepted 
recommendation for the use of different antimicrobials, neither for the treatment of peri-implant mucositis nor peri-implantitis (31). Antibiotics alone do not clean the surface, and there is growing concern that microbial resistance might render these agents inefficient in the long term. Guidelines on the use of antibiotics, mainly systemic, now only recommend them as a last resort against more severe cases of infections, but there is a lack of clinical evidence showing antibiotics have any effect at all on the long-term outcome of peri-implant conditions (32-34).

One of the recognised challenges for peri-implant treatment is accessibility. Aside from complicating local anatomy and bone defect morphology, prosthetic supra-structures often make it difficult to reach infected areas and thus prevent effective cleaning (20). Therefore, many of the traditional mechanical treatment interventions adopted from periodontics have their limitations due to accessibility $(12,20)$. Adaptation of the prosthetic design is essential for accessibility and continuity of peri-implant health $(12,20,35)$.

The present study aims to establish a common basis for future experimental studies and clinical trials in addition to benchmarking clinical tools in the handling of peri-implant diseases. The digital survey was distributed amongst experts skilled in dental implant-related clinical disciplines. It was designed to identify expectations and expected outcomes of commonly used procedures and to learn about the current obstacles and challenges in maintaining healthy peri-implant conditions. This survey represents an initial step towards establishing a common and more evidence-based clinical approach to managing peri-implant diseases.

\section{MATERIALS AND METHODS \\ Study Design}

The study followed an anonymised cross- sectional observational study design according to STROBE guidelines. Ethical approval is not required for this kind of surveys. A total of 70 experts from 16 countries, selected on referral from colleagues and by their contribution to relevant clinical literature, were invited by email to participate in the survey. The survey was kept open for participation from the beginning of October 2019 until the end of December 2019.

The results from the cross-sectional digital survey are based on semi-structured computer-assisted web interviews. Five pilot questionnaires were sent to and assessed by respondents to obtain feedback and finalise the structure of the survey. The survey was digitally administrated through NordiMed AS, which also collected, recorded, and sorted the answers. At NordiMed AS, answers were kept separated from email lists so that connexions between the names of respondents and their answers could not be made by the authors. The survey consisted of a set of pre-generated answers to be scored according to relevance and importance, a part that allowed participants to write free-text comments and suggestions on treatments, and a page open for general remarks and comments.

The survey consisted of 23 questions divided into seven subsections:
1. demographics, including primary workplace, age, education, what country they are working in, and how often they saw and treated patients with peri-implantitis;

2. critical criteria when choosing an implant debridement procedure/treatment;

3. product group perception, including experts' views on debridement procedures and remedies, as well as their clinical performance;

4. design input on present and future procedures and remedies, with a focus on chemical surface debridement and significant challenges to overcome;

5. the best mechanical cleaning/treatment procedure available today;

6. the scope and frequency of a peri-implant health support and maintenance programme;

7. open free-text boxes where participants were asked to write what procedures in peri-implant treatment they avoid and what factors they think are important for further improving management of implant-related conditions.

In section one, general information about the respondents was collected. In section two, the experts were asked to score the importance of ten criteria (defect anatomy, implant surface structure, general condition, oral condition, cleaning efficacy, short treatment time, low risk of damaging implant, mucosa or tooth, low risk of antibiotic resistance, low price, and welldocumented) on a fixed scale from zero to seven. In sections three, four, and five, participants were presented with key attributes from which to choose and rate in terms of importance ("predictability," "cleaning efficacy," "short treatment time," "low risk of damaging implant or mucosa," "low risk for antibiotic resistance," "low cost" and "well-documented"). Section five provided an opportunity for respondents to add other unlisted treatment option(s). In sections six and seven, respondents scored the preferred patient recall frequency and provided comments in free text.

\section{Data Analysis}

The data were analysed using descriptive statistics and are presented as percentages, absolute values, and medians with an interquartile range (IQR). Descriptive statistics are also presented for the following subset of groups: age, country, education, and workplace. Furthermore, correlation analyses were done between answers and sub-groups of respondents and between the different sets of answers. The scales were considered categorical; thus, the Spearman rank test was used for the correlation analysis. The Spearman's rho $\left(r_{s}\right)$ results were interpreted as follows: no correlation if $r_{s}<0.3$; correlation if $0.3<r_{s}<0.5$; and strong correlation if $0.5<r_{s}<1$ (36). A negative $r_{s}$ indicated a negative correlation, while a positive $r_{s}$ indicated a positive correlation. The qualitative part of the questionnaire was also evaluated to identify and present common standpoints and conflicting views. StataSE 16 (StataCorp, College Station, Texas USA) software was used for statistical analysis. For graphical figures, GraphPad Prism 8.3.0 software was used. 


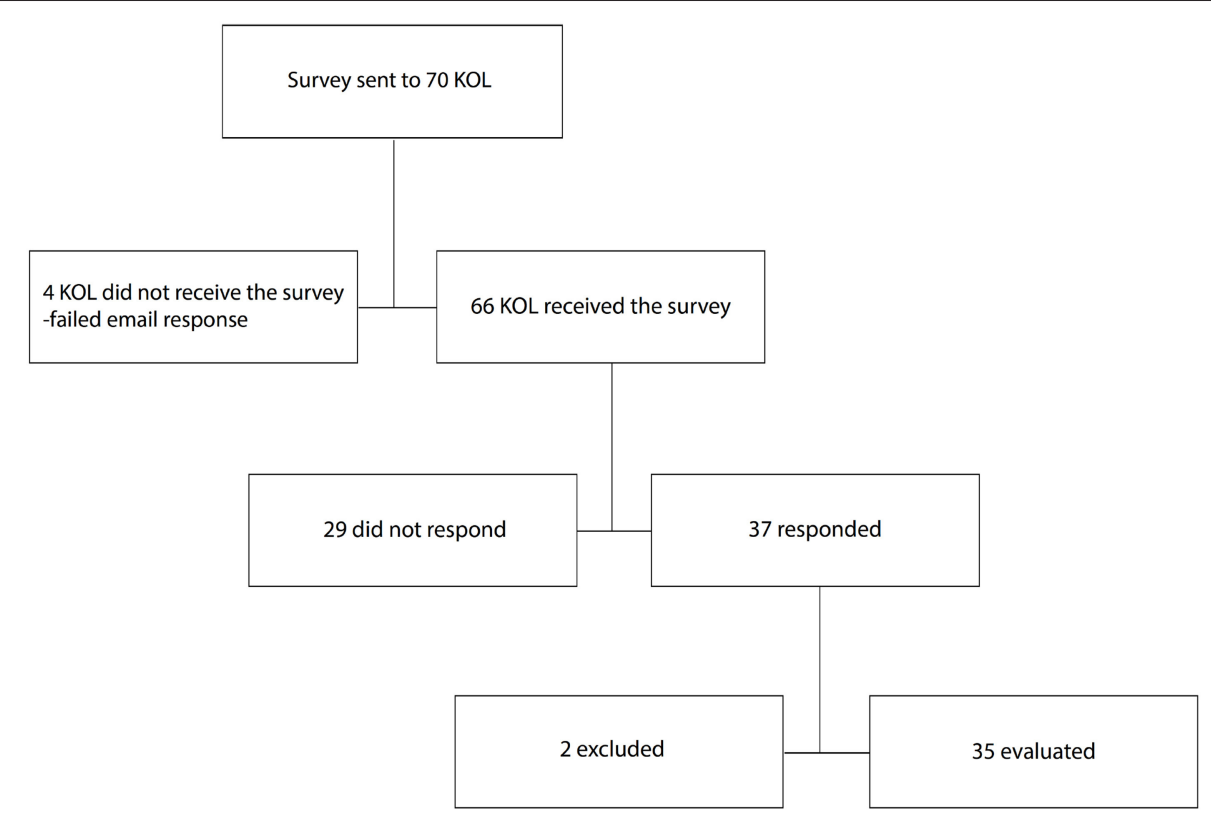

FIGURE 1 | Response diagram.

\section{RESULTS}

\section{Responses, Demographics, and Involvement in Treating Patients}

Sixty-six clinical experts received the survey (Figure 1); four were left out due to faulty or expired email addresses. By the end of December 2019, 37 of the invited experts had answered the survey (a 53\% response rate); two were excluded due to incomplete forms. A total of 35 complete responses from the invited experts were registered at the end of the survey period.

Education, primary workplace, age, and country of the respondents are disclosed in Figure 2. Eight percent of the participants were dentists holding a doctoral degree $(\mathrm{PhD})$ without a clinical speciality, $63 \%$ were dentists with both a clinical speciality and a $\mathrm{PhD}$ (a total of $71 \%$ of the participants had a $\mathrm{PhD}$ ). The remaining $29 \%$ were clinical specialists without a $\mathrm{PhD}$. Thirty-seven percentage of respondents' primary workplace was a private or public clinic ( $74 \%$ of the respondents jointly), while the remaining $26 \%$ of respondents' primary workplace was a university or university hospital.

Clinicians from 16 countries participated. Approximately $52 \%$ of respondents were from North-Western Europe, 34\% of respondents were from Southern Europe, and only $14 \%$ were not from Europe (division according to EuroVoc), the group outside Europe includes participants from US, Australia and Asia. The respondents' median age-range was $50-60$ years, with $69 \%$ of the respondents being older than 50 years (age range: $30-70$ years). All the respondents were directly involved in the treatment of peri-implant complications, and $74 \%$ reported seeing such patients often.

All 35 respondents highly recommended more RCTs on periimplant debridement procedures. Most respondents agreed that their answers could be used for benchmarking and developing procedures and wished to participate in future RCTs and clinical research projects. Only three respondents wanted to remain anonymous, and two did not want to participate in international initiatives in clinical research on peri-implant complications and implant health management.

\section{Choosing an Implant Surface Cleaning Procedure}

When choosing an implant surface debridement procedure, the criteria that scored as the most important were, in the order of reported significance, "cleaning efficacy" (7, IQR: 5-7) "welldocumented" (6, IQR: 5-7), "low risk of damaging implant surface, mucosa, or tooth" (6, IQR: 5-7) and "defect anatomy" (6, IQR: 5-7). "Low risk of antibiotic resistance" also scored high on importance (6, IQR: 4-7). Low price was considered the least important attribute (4, IQR: 3-5) (Figure 3). Regarding differences between groups, older respondents tended to consider documentation more important than those under 50 , but this difference was not statistically significant $(p=0.09)$. As for the other attributes, the respondents were in agreement.

\section{Views on Mechanical Treatment Options}

For mechanical debridement, brushes in motor-driven handpieces (34\%) and airflow and ultrasound devices (34\%) were the devices that obtained the highest scores for perceived efficacy (Figure 4). Implantoplasty was the third most popular mechanical treatment option (11\%) and was more popular among younger respondents (27\%). Together with airflow and ultrasound devices, implantoplasty was viewed as the most effective mechanical treatment option in this sub-group (Figure 5). For respondents over 50, implantoplasty was a 


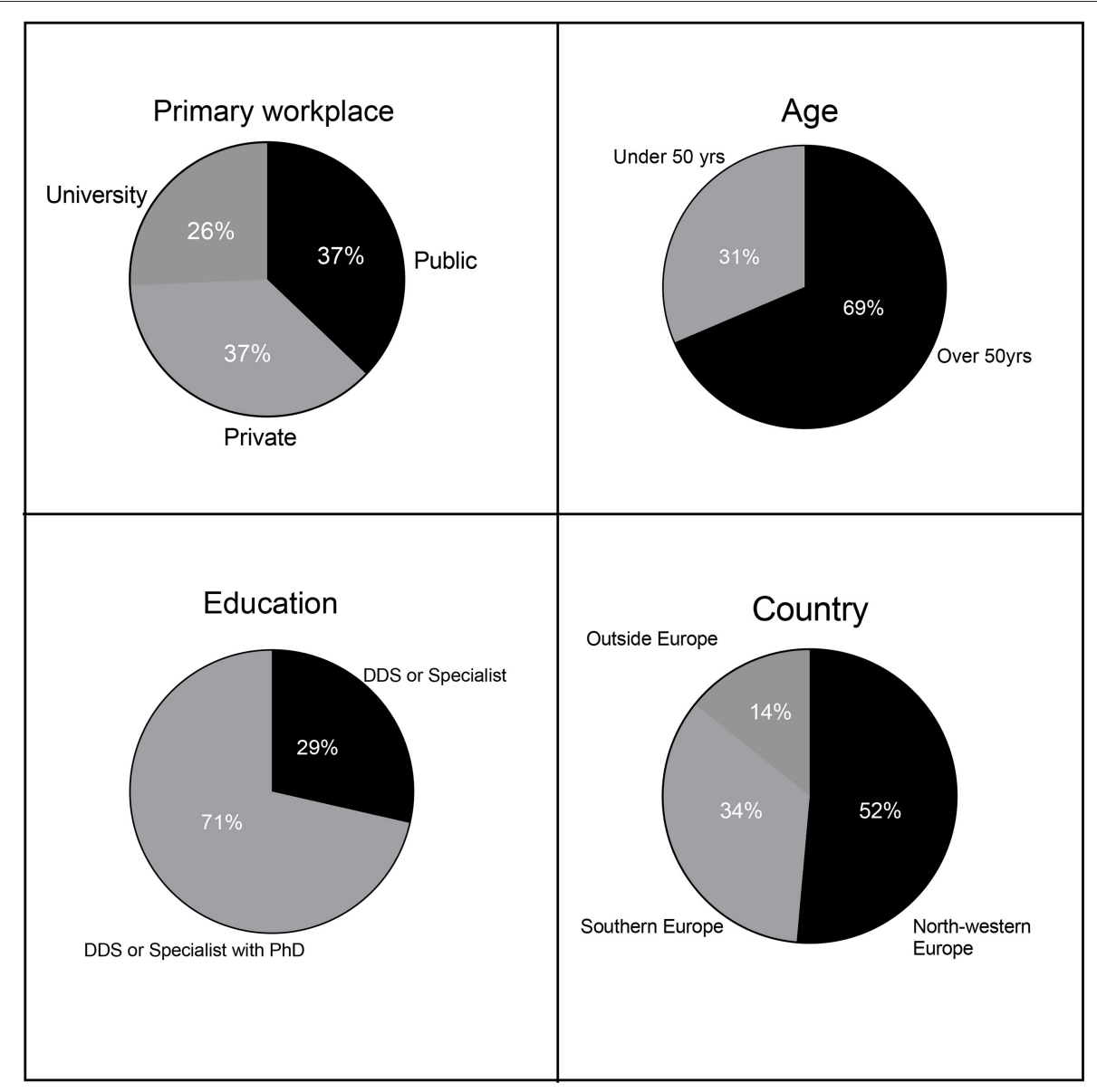

FIGURE 2 | Demography table.

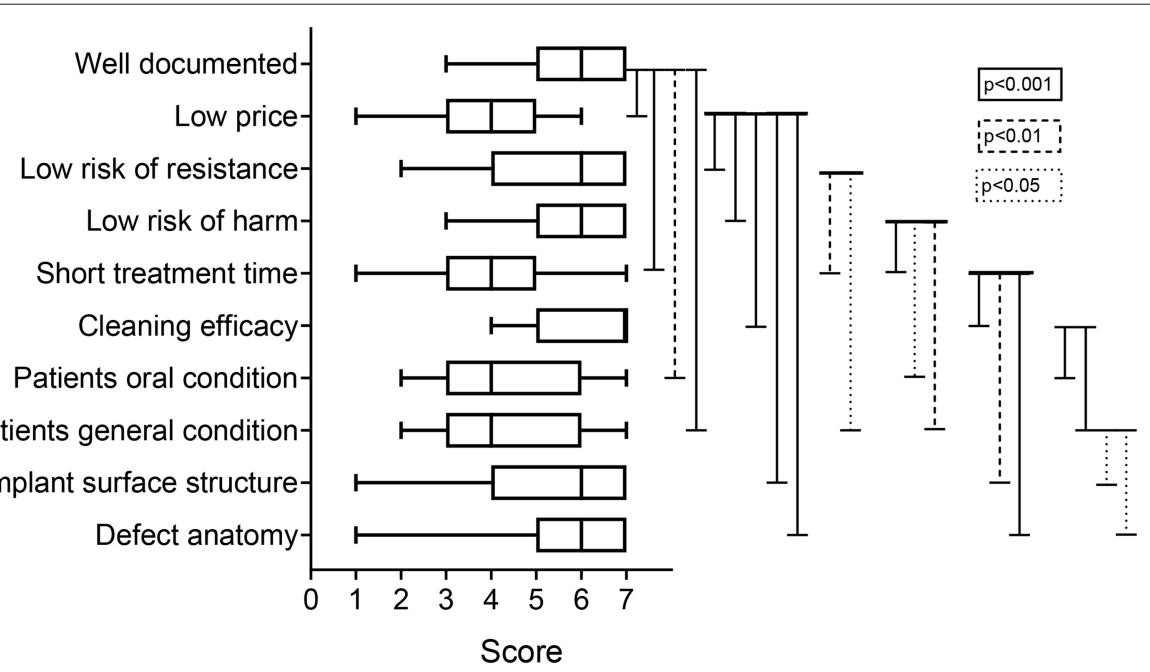

FIGURE 3 | Box-plot with score of importance of listed criteria when choosing implant-cleaning procedures. 


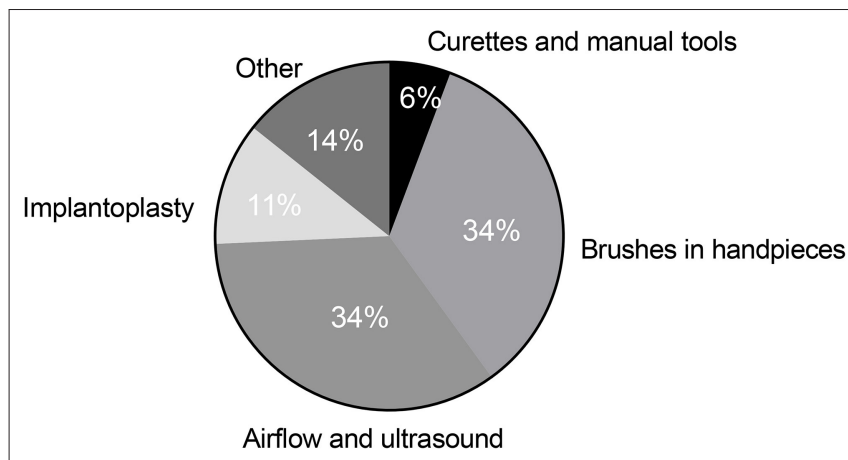

FIGURE 4 | Mechanical treatment preferences.

\section{Mechanical treatment preferences $<50 \mathrm{yrs}$}

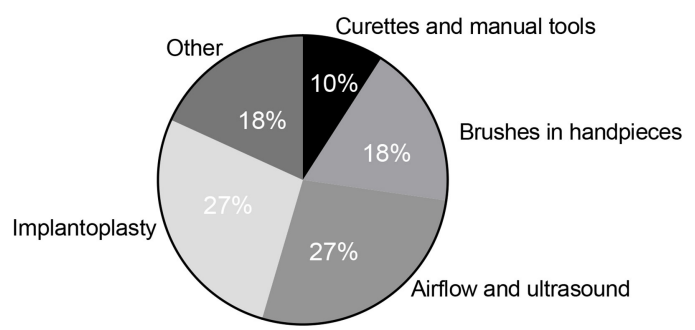

$n=11$

\section{Mechanical treatment preferences $>50 \mathrm{yrs}$}

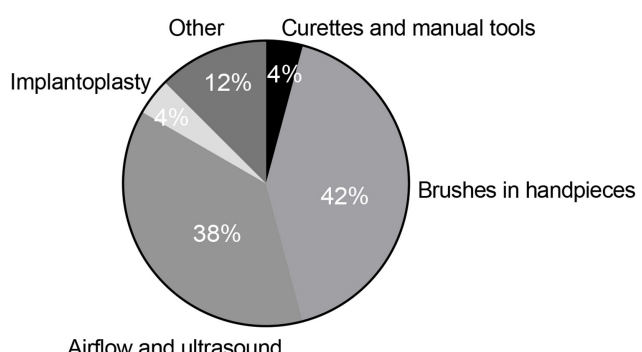

$n=24$

FIGURE 5 | Mechanical treatment preferences segmented by responder's age.

much less popular option; only $4 \%$ preferred this mechanical treatment. Brushes in motor-driven handpieces, airflow, and ultrasound devices remained the preferred mechanical treatment options among participants over 50 .
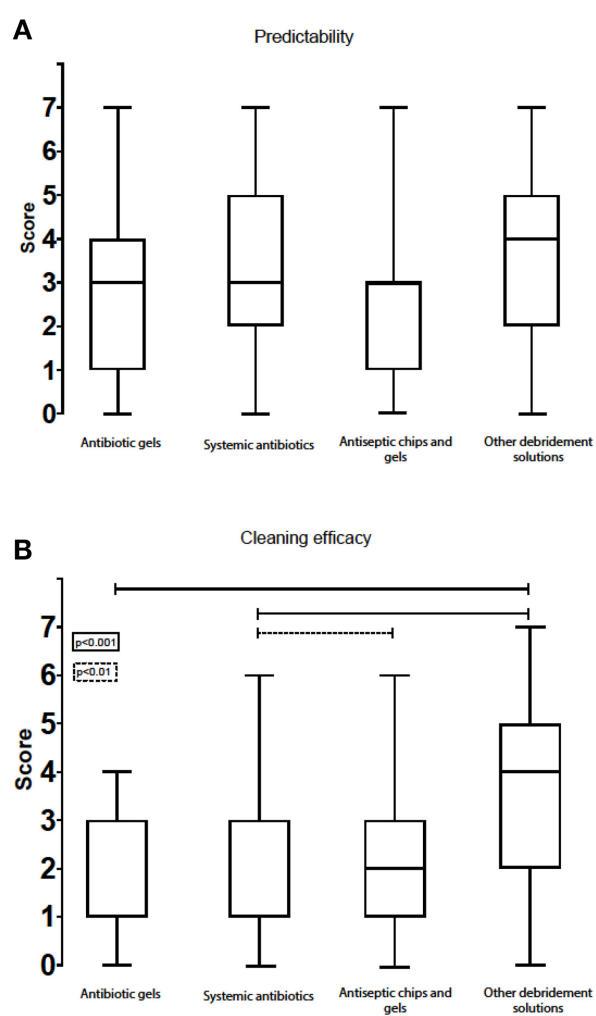

FIGURE 6 | Box-plot with score of predictability (A) and cleaning efficacy (B) for the various chemical remedies.

There were no statistical differences between geographical regions with respect to ratings of mechanical debridement procedures, nor were there any differences between clinical residents treating patients in private or public clinics or academic institutions.

\section{Chemical Remedies and Their Qualities Predictability}

As for the attribute "predictability," the option "other debridement solutions' (i.e., peroxides)" was considered the best option, with a median score of 4, IQR: 2-5. Systemic antibiotics came second (3, IQR: 2-5) and antibiotic gels third (3, IQR: 1-4). The lowest overall score was for antiseptic chips/gels (3, IQR: 1-3) (Figure 6A). When separating answers into sub-groups, there were only two differences: the younger subgroup tended to score higher on the predictability of systemic antibiotics than the older sub-group, and private practitioners considered "other debridement solutions" more predictable than did those working at public clinics or universities (Figure 7).

\section{Cleaning Efficacy}

When it comes to the efficacy of chemical debridement options, "other debridement solutions (i.e., peroxides)" scored best (4, IQR: 2-5). Antiseptic chips/gels scored lower (2, IQR: 1-3), while systemic antibiotics (1, IQR: 1-3) and antibiotic gels (1, IQR: 1-3) had the lowest medians on this attribute (Figure 6B). 


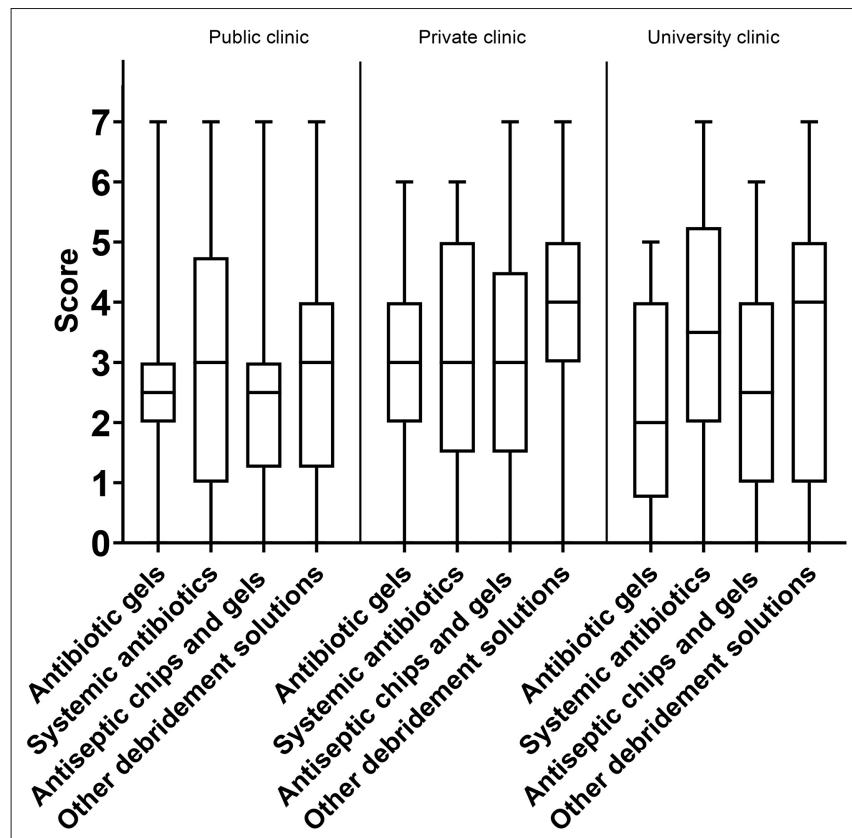

FIGURE 7 | Box-plot with score of predictability of chemical remedies segmented by responder's workplace.

\section{Treatment Time}

All four product groups listed in the survey scored similarly for "treatment time," with a median of five for all debridement solutions. However, the interquartile ranges varied significantly between antiseptic chips/gels (5, IQR: 2-5), systemic antibiotics (5, IQR: 2-7), antibiotic gels (5, IQR: 3-6), and other debridement solutions (5, IQR: 5-7) (Figure 8A). The option "other debridement solutions" showed an interquartile range that skewed more to the upper part of the scale and hence was considered to afford the shortest treatment time.

\section{Views on Harmful Effects}

Systemic antibiotics had the highest score on "low risk of damaging the implant surface, mucosa, or tooth" (7, IQR: 6-7). Antibiotic gels (6, IQR: 5-7) and antiseptic chips/gels (6, IQR: $5-7)$ were both one point lower. "Other debridement options" had the same median (6, IQR: 4-6) but a wider interquartile range in the lower part of the scale. None of the options scored a "poor performance."

\section{Awareness of Microbial Resistance to Antibiotics}

The experts scored systemic antibiotics very low under the attribute "low risk for inducing microbial antibiotic resistance" (1, IQR: 1-2). "Other debridement solutions" had the highest score (7, IQR: 6-7). Antiseptic chips/gels and antibiotic gels had a median score of 6, IQR: $3-7$ and 3, IQR: $2-5$, respectively (Figure 8B).
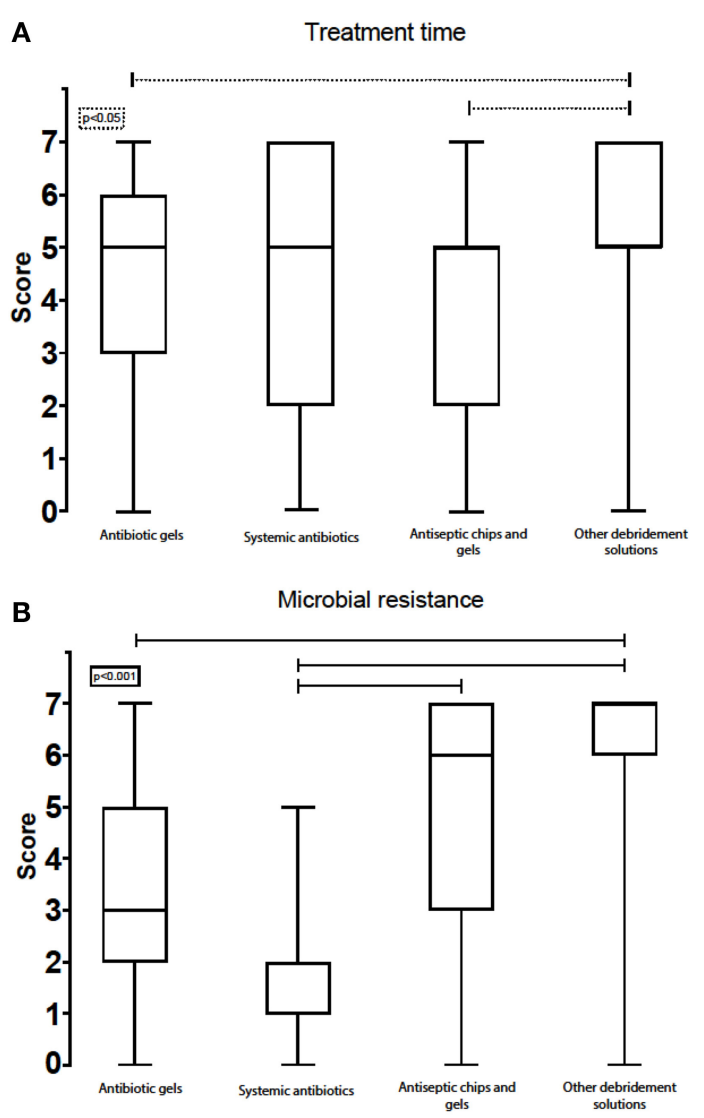

FIGURE 8 | Box-plot with score of treatment time (A) and microbial resistance (B) for the various chemical remedies.

\section{Importance of Direct and Indirect Treatment Costs}

Amongst the listed treatment options, "other debridement solutions" scored the highest on price vs. effect (7, IQR: 57). Systemic antibiotics came in second (6, IQR: 4-6), whereas antiseptic chips/gels was rated third (3, IQR: 1-4). Antibiotic gels scored as the most expensive treatment option (2, IQR: 1-3) (Figure 9A). Respondents working in private and public clinics had a tendency to report antibiotic gels as being less expensive than did experts working at universities (3, IQR: 1-3, 2, IQR: 1-3, 1, IQR: 0-2) (Figure 10). The same trend was seen for antiseptic chips/gels.

\section{Need for Published Clinical Evidence}

The scores of the clinical evidence and published documentation were best for systemic antibiotics (3, IQR: 2-5), followed by other debridement solutions (3, IQR: 2-5), antibiotic gels (3, IQR: 24 ), and antiseptic chips/gels (3, IQR: 2-4). All the scores were at the lower end of the scale. Systemic antibiotics and other debridement solutions had interquartile ranges that skewed more to the upper part of the scale and were thus regarded as treatment options with slightly better clinical evidence (Figure 9B). Private practitioners scored the available evidence for the use of systemic antibiotics higher than those working in public clinics and 


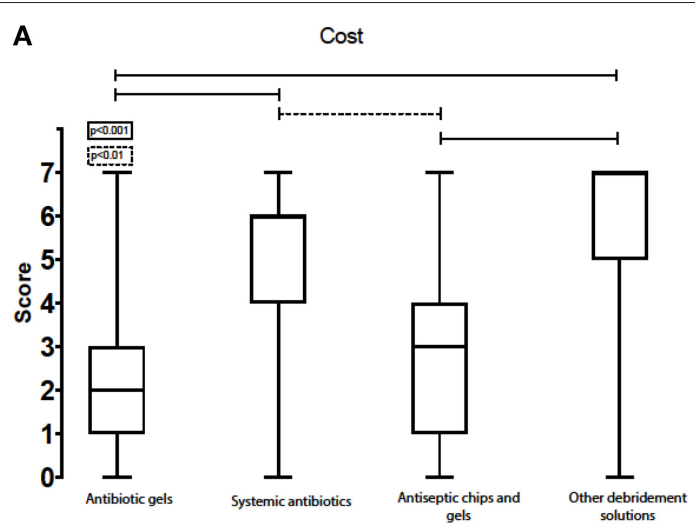

B Published clinical evidence

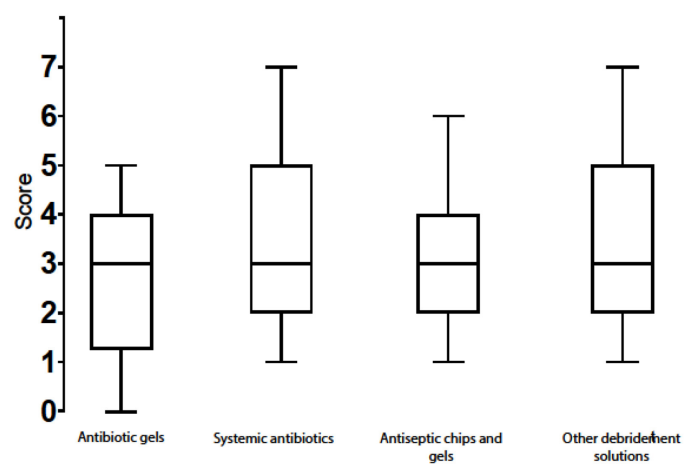

FIGURE 9 | Box-plot with score of cost (A) (lower numbers represent most expensive option) and published clinical evidence (B) for the various chemical remedies.

university hospitals (4, IQR: 3-6 and 3, IQR: 2-4, respectively). In addition, private practitioners scored local acting antibiotics as less documented than experts working at universities and public clinics (2, IQR: 2-3 and 3, IQR: 3-3, respectively).

\section{Need for New Remedies}

When asked for what they need and want from new therapies for peri-implantitis and peri-implant health maintenance, participants gave the following scores (listed in order of importance): eliminate risk for microbial resistance (7, IQR: 67); cleaning efficacy (6, IQR: 5-7); short treatment time (6, IQR: 5-7); safe, low risk of damaging implant surface, mucosa, or tooth (6, IQR: 5-7); and predictable treatment outcome (6, IQR: 4-7). Low cost and published clinical evidence had the lowest scores (4, IQR: 3-5 and 4, IQR: 2-6, respectively) (Figure 11).

\section{Views on Peri-Implant Health Maintenance Recall Frequency}

Regarding implant supportive treatments and maintenance programmes, there was significant agreement between participants on the recommended frequency of check-ups and professional rinsing and cleaning for patients with implant complications; $69 \%$ recommended regular maintenance
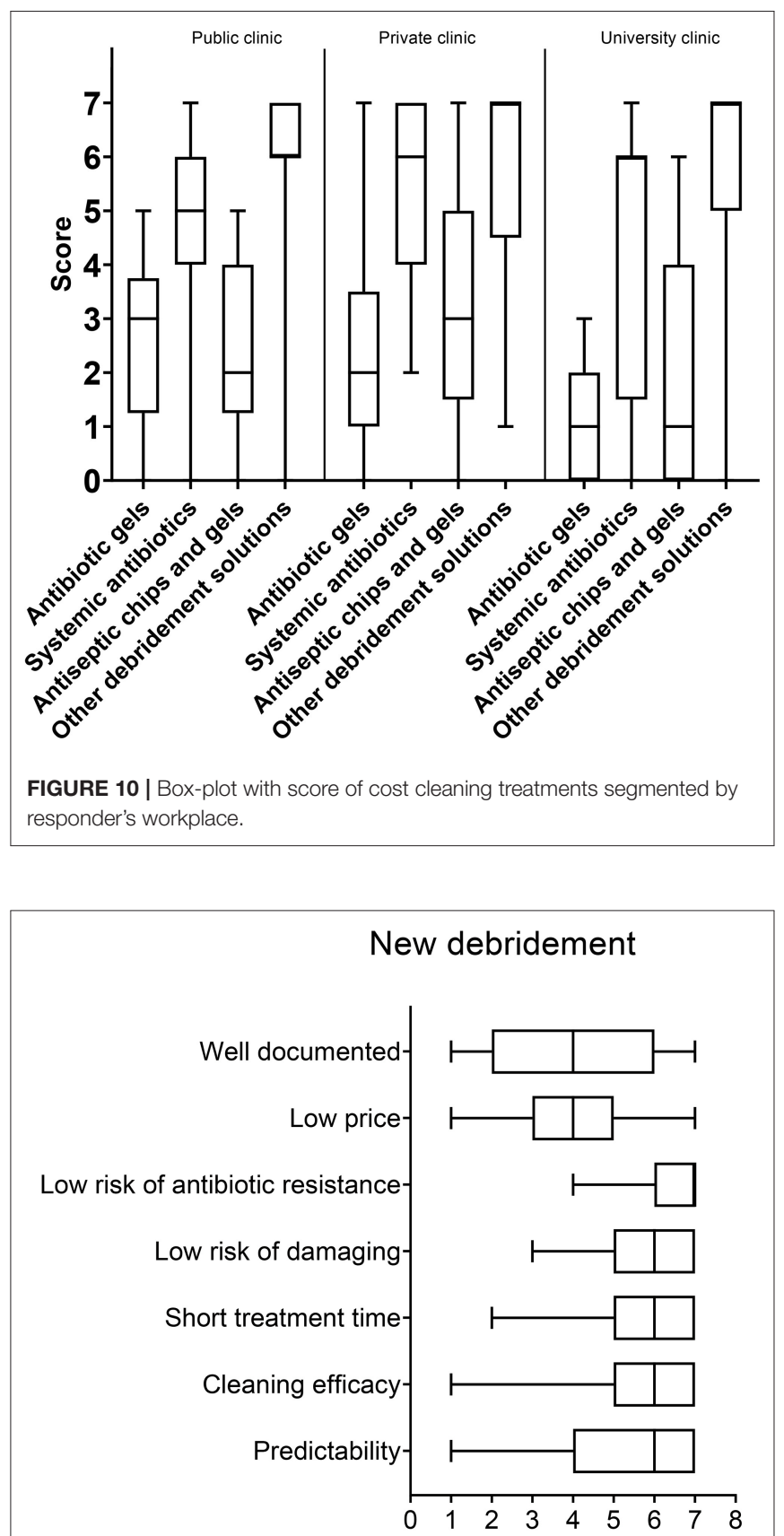

FIGURE 11 | Need from new therapies for peri-implantitis and peri-implant health maintenance.

treatment three times annually or more, while $23 \%$ thought that twice annually is sufficient, and only $8 \%$ thought that once annually is adequate.

\section{What to Avoid When Treating Peri-Implant Complications}

In the last part of the survey, participants were asked to write, based on experience, in free text, if any procedure or 
remedy in dentistry should be avoided when treating periimplant complications. They were also asked to elaborate on their recommendations. As many as 12 experts stated that implantoplasty should be avoided. When merging all mechanical debridement procedures (metal curettes, rotating brushes, plastic curettes, air abrasive, plastic tips, and implantoplasty), 24 of the 35 respondents stated that the use of these remedies should be avoided or at least minimised to the bare necessity. Moreover, 10 of the experts thought that the use of antibiotics should be avoided altogether. In contrast, 13 of the respondents answered that none of the available treatments and debridement procedures should be avoided entirely and should all be applied on an individual basis.

\section{Correlations Between Groups and Views}

When analysing the answers, correlations between groups and views became evident. For example, defect anatomy was regarded as the patient criterion that most significantly impacts the treatment outcomes of peri-implantitis procedures. The experts who saw the size, shape and location of the bone defect (aka "bone defect anatomy") as the critical factor for choosing treatment also thought that patients' general and oral health were crucial factors to consider $\left(\mathrm{r}_{\mathrm{s}}=0.44, p<0.01\right)$.

Participants who regarded cleaning efficacy as the critical factor to consider when choosing a procedure also considered the safety of the debridement procedure very important $\left(\mathrm{r}_{\mathrm{s}}=0.60, p\right.$ $<0.01$ ). There was no significant correlation between those who saw chemical debridement efficacy and safety as essential factors and those who wanted to avoid implantoplasty.

When respondents were divided into groups by region (Figure 2), there were differences in their views on the safety and efficacy of antibiotics. Although not statistically significant, clinicians from North-Western Europe were more concerned about microbial resistance than those from Southern Europe and outside Europe (1, IQR: 1-2 and 2, IQR: 1-2, respectively). Experts from Southern Europe seemed to regard systemic antibiotics as safer than did North-Western Europeans. When opting for an improved implant debridement solution, clinicians from North-Western Europe more strongly emphasised the need to avoid microbial resistance (6, IQR: 5-7 vs. 5, IQR: $4-6$, respectively).

A significant correlation between the attributes "short treatment time" and "low price" was observed $\left(\mathrm{r}_{\mathrm{s}}=0.58, p<0.01\right)$.

\section{DISCUSSION}

Awareness and knowledge about the biological, mechanical, and clinical causes of peri-implant diseases, how to diagnose them, how to treat with a predictable outcome, and the prognosis for the diseases have improved significantly in recent years. However, after numerous papers and reports on peri-implant ailments and health, there appears to be no consensus among clinicians and researchers on these topics. There also seem to be regional differences in how the problem is conceived of and dealt with. Studies evaluating clinicians' opinions regarding implant dentistry and the now well-known complication periimplantitis have previously been reported in the literature (3739). Results from these studies vary. One recent survey on implant dentistry, including a section with peri-implantitis using the Delphi method, was used to assess the potential trends for the year 2030 in implant dentistry in Europe (40). Experts in implant dentistry reached a consensus regarding peri-implantitis, namely that there will be an increase in the incidence of periimplantitis in the future, adding that there will be more efficient interventions in the form of treatment and prevention (40). Today, various tools are available for the treatment of periimplant complications. However, no single therapy is considered satisfactory or superior, as reflected in the results of this study.

Cleaning efficacy was clearly important to the experts. However, all agreed that cleaning efficacy should be safe and not harmful to implant surfaces or tissues. Participants also considered proper clinical documentation to be of crucial importance. These answers bring out some of the main challenges in peri-implant treatments, such as the need for an effective and well-documented but not harmful treatment option. The criterion "low risk for inducing microbial antibiotic resistance" also scored high. The use of antibiotics was generally not recommended, even though it was viewed as a low-cost, noninvasive, and moderately effective alternative. Low costs are generally not regarded as important in peri-implant treatments, indicating that experts do not tend to opt for the cheapest alternatives. On the contrary, there is a willingness to use effective, predictable, and well-documented treatment options regardless of cost and chair-time. A qualitative report showed that patients suffering from periodontitis are willing to invest time and financial resources to become healthy, especially since lacking teeth is regarded as socially limiting in the industrialised part of the world (41). Patients who have already invested in implants to replace missing teeth and develop peri-implantitis might be even more concerned with their disease than periodontitis patients. They have already spent a significant amount of time and money on treatment, and they are probably willing to pay more to keep their implants healthy, which again affects clinicians' views on the importance of cost-effectiveness. Peri-implant disease treatment is thus likely less price sensitive than other treatments, allowing clinicians to focus more on safety, efficacy, predictability, and long-term treatment outcomes.

In the present study, surgical and non-surgical treatments were not separated. The effect of mechanical and chemical debridement independent of open or closed access was evaluated. Unlike other treatment modalities, motor-driven brushes are developed solely for peri-implant disease management and are frequently used and were highly valued amongst the responding experts. Airflow and ultrasound devices attained scores equal to motor-driven brushes and were also regarded as treatment options providing good results. However, studies evaluating these treatment options are scarce $(42,43)$. This is also reflected in the present survey, as expert responses were divided into four equal groups based on their opinions regarding the best currently available mechanical treatment option. Implantoplasty gravely reshapes the structure, chemistry, and wettability of the 
implant's surface (44). Even though this treatment option had the third highest score, in the free-text part of the survey, twelve participants argued that implantoplasty should be avoided. Accordingly, there are conflicting opinions regarding the use of implantoplasty as a treatment option, which is also reflected in the current clinical literature $(19,26,27,29,30,44)$.

Since the survey was performed before the COVID-19 pandemic, none of the respondents brought up the issue of aerosol formation and virus transfer as a safety issue during clinical procedures in the dental clinic. Opinions may since have shifted, as procedures such as air-blasting, ultrasound devices, and turbine drills, all of which pose a risk of producing contaminated aerosols (45), are to be reduced as much as possible.

From the median scores and interquartile ranges on predictability, it is evident that no particular debridement solution is superior. The interquartile ranges were wide, and no debridement solution presented a median score over four, indicating that none of the evaluated treatment options were considered predictable by the responding experts. There was strong agreement amongst the experts that there is an urgent need for new, non-antibiotic cleaning options with predictable outcomes that can be used as adjuncts to mechanical debridement.

Cleaning efficacy was considered the most crucial factor when choosing an anti-microbial/anti-fouling debridement option. Three debridement options scored low on this attribute. Even the treatment option with the highest score had only a medium value, suggesting that the experts believe none of the available chemical debridement solutions performs satisfactorily when it comes to cleaning efficacy. There are also good reasons that antibiotics scored low on this attribute. The interpretation of cleaning effectiveness is considered to be the level of disruption of the biofilm and the removal of the biofouling from the implant surface by the use of chemical and/or mechanical remedies. Systemic antibiotics are not very good at this, since they only kill microbes or restrict their growth without physically removing or dissolving them $(46,47)$. Systemic antibiotics are also inherently poor at penetrating biofilms $(48,49)$. Local antibiotics are considered neither to disrupt the biofilm nor remove the fouling, but only control bacterial (re-)growth (50). Other debridement solutions like peroxides and chlorine scored the highest; the IQR was wide and skewed to the lower part of the scale. Cleaning efficacy was the most important criterion amongst the experts. However, this criterion had the overall lowest scores for the presently available chemically debridement options, highlighting that new safe, effective, and predictable chemical debridement options are still in high demand.

Regarding treatment time, all debridement solutions had a median score at the upper part of the scale, meaning that none of the debridement solutions was considered to have a long treatment time. However, antiseptic chips, systemic antibiotics, and antibiotic gels had wide interquartile ranges, which indicates divided opinions regarding treatment time and efficacy. This might be due to different procedures being followed by the experts and a lack of general agreement amongst experts on procedures for treating peri-implantitis. However, a short treatment time was not considered an important criterion when choosing debridement procedures (4, IQR: 3-5). Nevertheless, participants pointed out the important interrelationship between ease of use, treatment time, and effectiveness-not only in terms of patients' well-being, but also for the clinicians' satisfaction. It should be possible for general clinicians without excessive implantology training to perform the treatment easily and predictably.

There was general agreement amongst the experts on the relatively low risk of harm to local structures/tissues from these procedures and remedies. This was especially evident for systemic antibiotics, which have little physical impact on implant surfaces, and mature teeth and are rigorously tested for safety. This attribute is important when choosing a debridement procedure, so the general agreement that available chemical debridement options are mostly harmless to the implant surface, mucosa, and teeth indicates that the participants agreed that none of these options should be excluded from use for peri-implant safety reasons.

The experts all agreed that the risk for inducing antibiotic resistance in microbes is highest when systemic antibiotics are used. This is an attribute that is considered vital when choosing an implant debridement solution. The participants all wished to avoid debridement procedures that can induce antibiotic resistance in microbes. Antibiotic resistance is linked to increased risk of morbidity, more prolonged hospitalisations, increased risk of treatment failure, and other serious complications (51). The use of antibiotics in dentistry can significantly contribute to this load, as bacteria from oral infections are frequently known to enter the gut and bloodstream (52-54). Studies have also shown that the submucosal pathogens from peri-implant patients frequently develop resistance to multiple antibiotics (55). Thus, the importance of not contributing unnecessarily to this harm by using fewer antibiotics in general is clear, as also indicated by the scoring from experts partaking in this survey. Nevertheless, a recent study from Norway on prescription of antibiotics showed that antibiotic therapy is more commonly used for treating peri-implantitis than periodontitis (56). In the free-text part of this survey, 10 of the 35 respondents stated that they avoid using systemic antibiotics entirely and see the development of microbial resistance as a serious clinical problem in dentistry. However, antibiotics are still used extensively to treat these pathologies (57).

The observed regional differences in views on antibiotics might be a reflection of the more profound load of multidrug resistant microorganisms in the southern and eastern parts of Europe (58). The more liberal use of antibiotics in these parts of Europe has been reported previously and probably mirrors differences in sociodemographic factors, patient expectance, and public healthcare availability between the regions (59). As the problem of microbial resistance towards antibiotics is progressing, the differences are likely to disappear simply because the antibiotic drugs will stop working and will need to be replaced by other remedies.

Opinions are more divided on using local acting antibiotic gels, showing a median score on the lower part of the scale, but with a relatively wide interquartile range. Some 
participants regarded antibiotic gels as contributors to microbial antibiotic resistance, while others did not. Regarding antiseptic chips, there was also disagreement on the risk of inducing resistance; however, the median score here was higher. The interquartile range was shifted towards the lower end of the scale, again suggesting that the respondents disagreed on how much these treatment options contribute to microbial resistance against antibiotics.

In contrast, all the experts agreed that "other debridement solutions" had a very low risk of inducing microbial resistance (7, IQR: 6-7). Notably, all respondents, even though they may have disagreed about the details, saw microbial resistance as a significant problem that has to be contained. Overall, reducing the use of antibiotics is an issue of global importance.

The experts agreed that antibiotic gels are the most expensive treatment option today. They were more divided on the cost effectiveness of the other debridement options. The cost of the treatment options listed seem, at least in part, related to what country the experts were practising in, perhaps reflecting the influence of local health insurance policies. Pricing is of some importance, both for the clinician and patient when choosing treatment options, as discussed earlier. However, experts participating in this survey mostly agreed that treatment costs, both in time and money, are of less importance when treating peri-implantitis. The costs involved have little influence on the choice of treatment and remedies for this serious condition.

The experts were not satisfied with the quality and quantity of the available scientific evidence for the various treatment options against peri-implant complications. Even though none of the chemical debridement options scored high on documented effects, private practitioners scored the available evidence for the use of systemic antibiotics higher than did those working at public clinics or university hospitals (4, IQR: 3-6 and 3, IQR: $2-4$, respectively). Interestingly, the same private practitioners scored local acting antibiotics as less documented than experts working at university and public clinics (2, IQR: 2-3 and 3, IQR: 3-3, respectively). This variance in opinions may in part be due to known variations in short- and long-term effects from adjunctive antibiotics use (60) and may also reflect the referral of persistent or difficult cases to the public and university clinics. Another explanation is that experts in public and academic institutions are less concerned with efficient time schedules and patient turnover. Private clinics, conversely, might lean more towards efficient routines. The interquartile range was somewhat larger for "systemic antibiotics" and "other debridement options," suggesting disagreement about the evidence for these remedies. None of the experts considered the application of chemical debridement solutions to be welldocumented, which highlights the need for new controlled clinical trials and clinical comparative studies. This is also reflected in the experts' willingness to contribute to international research initiatives.

The experts' positive attitudes towards new debridement remedies are a good indication of the real and pressing need for new, safe, and more effective treatment options for peri-implant complications. Documented clinical evidence was considered an essential factor amongst the participating clinical experts.
Still, they did not expect a new procedure or remedy to be very well-documented when first introduced. This was not very surprising, as evidence for clinical effectiveness and safety in dental implantology includes long observation times and typically years of follow-up, as evidence of the effectiveness of new procedures and devices must be accumulated over time. The answers indicate that the experts saw an urgent need for new and improved therapies customised for implants and not just adapted from periodontal treatment. There was an explicit willingness to test new procedures and remedies and participate in optimising protocols for peri-implantitis treatment and implant health maintenance and contribute to documentation of the efficacy and safety of such new devices and procedures.

All the experts agreed that maintenance programmes should be initiated for patients with peri-implant complications. The numbers support findings in previously published studies (61-63) and underline the need for close follow-up of implant patients. Regarding maintenance programmes, the participants stressed that individual factors such as poor plaque control, previous periodontal disease, and smoking (61-63) should be taken into consideration. Several respondents highlighted the importance of individually customised maintenance programmes and generally having a high-frequency recall schedule for patients with peri-implant ailments. Studies on this specific theme have suggested that regular maintenance increases the likelihood of continued peri-implant health (61). Still, there is no general agreement on protocols for maintenance methods and frequency in the literature other than that it should be "frequent and individualised" (62). Designing and developing a risk assessment tool might be useful for identifying individuals at risk and making it easier to predict the recall interval (64), risk assessment has been used extensively for periodontal disease (65).

Participants raised several issues about standardised protocols for clinical intervention against peri-implantitis and maintenance of peri-implant health. The topics most frequently discussed (by nine of the 35 experts) were the importance of the prosthetic construction and access (both for clinician and patient) to the (exposed part of the) implant; the possibility to remove, adjust, and modify the supra-constructions; and the possibility to keep the prostheses clean. Concerns about the biocompatibility of the materials used and how the materials themselves contribute to the inflammatory conditions were also raised. Some experts mentioned the pain and discomfort caused by the present cleaning procedures as a cause for no-shows and a lack of patient compliance to maintenance programmes. The need for education and training for general dentists and hygienists on how to examine peri-implant tissues, diagnose peri-implant complications and how to handle implant maintenance programmes was also a common concern. Lastly, several of the participants raised the issue of individualising maintenance programmes according to the brand of implant (or implant surface) and called for implant manufacturers to provide evidence-based information on maintenance recommendations for the various implants on the market.

Experts who regarded cleaning efficacy as the critical factor to consider when choosing a procedure also considered the 
safety of the debridement procedure to be very important. This suggests that these respondents want debridement methods with high cleaning efficacy that are also harmless to surfaces and tissues. This is not feasible with established, available options for chemical implant debridement today (even though most of the experts scored these remedies as mostly safe, they also scored them as ineffective), as they are either aggressive and/or toxic (peroxides, chlorides, alcohols, etc.) or ineffective at removing fouling and contamination from the surfaces (antibiotics, antiseptics, etc.), which demonstrates the need for new, improved remedies for safe, effective in situ chemical surface cleaning.

Intriguingly, there was no significant correlation between those who saw chemical debridement efficacy and safety as important factors and those who wanted to avoid implantoplasty. This might imply that these experts did not regard implantoplasty as a debridement procedure per se, but rather as a last mechanical resort to try to rescue contaminated, ailing implants, making the surface smoother and easier to clean during the following implant maintenance phase. Implantoplasty is then not aimed at saving the implant surface and structure, which is forever lost during the procedure, but rather at saving the implant and improving the cleanability of the complete prosthetic construction. This difference in treatment aim-save the implant or save the overall (prosthetic) function-can be conflicting and impact the choice of treatment protocols. To improve the quality of future studies, it will be necessary to better define treatment aim(s) to the participating clinicians so that they have a common understanding of the problem and can benchmark objectives and align their strategies and protocols to make scoring of treatment outcomes more comparable across study arms and trials.

The experts who think cost-effectiveness is important also consider fast treatment and low costs as critical clinical issues when treating patients with peri-implant complications. This can arguably be in the interest of patients and motivate patient compliance in recall schemes. As the literature has shown, patients influence treatment procedure decisions, and they are influenced by cost, time, and their relationship with their dentist (66). Respondents who considered these factors important might also have the acutely ill patient's interest in mind since fast and low-cost treatments are often easier to implement ad hoc in clinics that see acute cases. However, we did not find any significant correlation to workplace (private, public, or academic) regarding these criteria. This suggests that the majority of the participating clinicians had a steady influx of patients with peri-implant complications, and that concerns about price and treatment time were actually more related to patient compliance with maintenance programmes and available chair-time. This might then actually be an indirect indicator for the (large) scale of the implant health management challenge.

The use of recognised experts in the field and the geographical distribution contributed to the strength of the present study. One of its limitations was the small number of participants and it can be discussed whether this number is representative for the majority of specialists in the field. Nevertheless, the response rate was 53\%, which indicates that those interested in the field and dealing with these issues were approached and willing to participate. Implants and implant surfaces are different from tissues and teeth and materials used in other dental disciplines and therefore need to be treated otherwise. Most implants used today are made of titanium or titanium alloys, and many also have their surfaces modified to improve their functions and effects on the adjacent tissues, especially alveolar bone. When installed, dental implants also have activated surfaces that are different from the bio-inert materials used in restorative dentistry. This is an important factor when considering peri-implant treatment strategies. This survey shows that there is still disagreement amongst clinical experts concerning such treatment choices, the factors that impact their choices, and the treatment aims and expected outcomes. For future evaluation, the different approaches for peri-implant mucositis treatment, and treatment choices based on caseto case and evaluation of the more complicated procedures is of interest. There is agreement amongst the experts who responded to this survey about the urgent need for more controlled clinical studies, with sufficient follow-up periods. There was overwhelming willingness amongst the experts to participate in such studies, underlining the need for clinical research on peri-implant complications and the establishment of standardised clinical protocols. A common observation was that current treatment procedures and debridement remedies give varying results depending on the type of implant, patient condition, and clinical protocols, and none of the treatment outcomes is predictable and completely satisfying. The experts agreed that there is a need to develop new, safe, and effective procedures and remedies against peri-implant diseases, specifically designed to work with implants and not based on antibiotics. They also agreed that patients treated for periimplant complications should be included in life-long implant health maintenance programmes with regular recalls two to four times annually.

\section{DATA AVAILABILITY STATEMENT}

The raw data supporting the conclusions of this article will be made available by the authors, without undue reservation.

\section{ETHICS STATEMENT}

Ethical review and approval was not required for the study in accordance with the local legislation and institutional requirements. The participants provided their written informed consent to participate in this study.

\section{AUTHOR CONTRIBUTIONS}

SL, HH, MS, and AA conceived the idea and prepared the survey. SL, BH, and $\mathrm{HH}$ collected the data. $\mathrm{BH}, \mathrm{SL}$, and $\mathrm{HH}$ analysed the data. $\mathrm{BH}, \mathrm{HH}, \mathrm{MS}, \mathrm{AA}$, and SL led the 
writing. GA, PB, DB, PE, KJ, SJ, EK, BK, CN, PP, AP, SR, JS-E, ASp, ASt, AV, and DV contributed with input to the survey, reviewed the data, and participated in writing of the manuscript. All authors contributed to the article and approved the submitted version.

\section{REFERENCES}

1. Levignac J. Periimplantation osteolysis- periimplantosis - periimplantitis. Rev Fr Odontostomatol. (1965) 12:1251-60.

2. Mombelli A, van Oosten MA, Schurch E Jr, Land NP. The microbiota associated with successful or failing osseointegrated titanium implants. Oral Microbiol Immunol. (1987) 2:14551. doi: 10.1111/j.1399-302X.1987.tb00298.x

3. Zitzmann NU, Berglundh T. Definition and prevalence of periimplant diseases. J Clin Periodontol. (2008) $35 \quad$ (8 Suppl.):28691. doi: 10.1111/j.1600-051X.2008.01274.x

4. Renvert S, Persson GR. Periodontitis as a potential risk factor for peri-implantitis. J Clin Periodontol. (2009) 36 (Suppl. 10):9-14. doi: 10.1111/j.1600-051X.2009.01416.x

5. Lindhe J, Meyle J, Group DoEWoP. Peri-implant diseases: consensus report of the sixth European workshop on periodontology. J Clin Periodontol. (2008) 35 (8 Suppl.):282-5. doi: 10.1111/j.1600-051X.2008. 01283.x

6. Lang NP, Berglundh T, Working Group 4 of Seventh European Workshop on P. Periimplant diseases: where are we now?-Consensus of the seventh European workshop on periodontology. J Clin Periodontol. (2011) 38 (Suppl. 11):178-81. doi: 10.1111/j.1600-051X.2010.01674.x

7. Tonetti MS, Chapple IL, Jepsen S, Sanz M. Primary and secondary prevention of periodontal and peri-implant diseases: introduction to, and objectives of the 11th European workshop on periodontology consensus conference. J Clin Periodontol. (2015) 42 (Suppl. 16):S1-4. doi: 10.1111/jcpe. 12382

8. Jepsen S, Berglundh T, Genco R, Aass AM, Demirel K, Derks J, et al. Primary prevention of peri-implantitis: managing peri-implant mucositis. $J$ Clin Periodontol. (2015) 42 (Suppl. 16):S152-7. doi: 10.1111/jcpe.12369

9. Tozum TF, Dursun E, Galindo-Moreno P, Juodzbalys G, Lopez-Martinez J, O'Valle F, et al. The 1(st) Baltic osseointegration academy and lithuanian university of health sciences consensus conference 2016. Summary and consensus statements: group II - peri-implantitis diagnostics and decision tree. J Oral Maxillofac Res. (2016) 7:e11. doi: 10.5037/jomr.2016.7311

10. Heitz-Mayfield LJ, Aaboe M, Araujo M, Carrion JB, Cavalcanti R, Cionca $\mathrm{N}$, et al. Group 4 ITI consensus report: risks and biologic complications associated with implant dentistry. Clin Oral Implants Res. (2018) 29 (Suppl. 16):351-8. doi: 10.1111/clr.13307

11. Berglundh T, Armitage G, Araujo MG, Avila-Ortiz G, Blanco J, Camargo PM, et al. Peri-implant diseases and conditions: consensus report of workgroup 4 of the 2017 world workshop on the classification of periodontal and periimplant diseases and conditions. J Periodontol. (2018) 89 (Suppl. 1):S313S8. doi: 10.1002/JPER.17-0739

12. Renvert S, Hirooka H, Polyzois I, Kelekis-Cholakis A, Wang HL, Working G. Diagnosis and non-surgical treatment of peri-implant diseases and maintenance care of patients with dental implants - consensus report of working group 3. Int Dent J. (2019) 69 (Suppl. 2):12-7. doi: 10.1111/idj.12490

13. Araujo MG, Lindhe J. Peri-implant health. J Clin Periodontol. (2018) 45 (Suppl. 20):S230-S6. doi: 10.1111/jcpe.12952

14. Ephros H, Kim S, DeFalco R. Peri-implantitis: evaluation and management. Dent Clin North Am. (2020) 64:305-13. doi: 10.1016/j.cden.2019.11.002

15. Derks J, Schaller D, Hakansson J, Wennstrom JL, Tomasi C, Berglundh T. Peri-implantitis - onset and pattern of progression. J Clin Periodontol. (2016) 43:383-8. doi: 10.1111/jcpe.12535

16. Koldsland OC, Wohlfahrt JC, Aass AM. Surgical treatment of peri-implantitis: prognostic indicators of short-term results. J Clin Periodontol. (2018) 45:10013. doi: $10.1111 /$ jcpe. 12816

\section{FUNDING}

This work was supported by a Project Establishing Support (PES) grant no. 328903 from the Research Council of Norway to SL.

17. Lee CT, Huang YW, Zhu L, Weltman R. Prevalences of peri-implantitis and peri-implant mucositis: systematic review and meta-analysis. J Dent. (2017) 62:1-12. doi: 10.1016/j.jdent.2017.04.011

18. Esposito M, Grusovin MG, Worthington HV. Treatment of peri-implantitis: what interventions are effective? A Cochrane systematic review. Eur J Oral Implantol. (2012) 5 (Suppl.):S21-41. doi: 10.1121/1.39986

19. Khoury F, Keeve PL, Ramanauskaite A, Schwarz F, Koo KT, Sculean A, et al. Surgical treatment of peri-implantitis - consensus report of working group 4. Int Dent J. (2019) 69 (Suppl. 2):18-22. doi: 10.1111/idj.12505

20. Renvert S, Polyzois I. Treatment of pathologic peri-implant pockets. Periodontol 2000. (2018) 76:180-90. doi: 10.1111/prd.12149

21. Lin GH, Suarez Lopez Del Amo F, Wang HL. Laser therapy for treatment of peri-implant mucositis and peri-implantitis: an American Academy of Periodontology best evidence review. J Periodontol. (2018) 89:76682. doi: 10.1902/jop.2017.160483

22. John G, Sahm N, Becker J, Schwarz F. Nonsurgical treatment of periimplantitis using an air-abrasive device or mechanical debridement and local application of chlorhexidine. Twelve-month follow-up of a prospective, randomized, controlled clinical study. Clin Oral Investig. (2015) 19:180714. doi: 10.1007/s00784-015-1406-7

23. Wohlfahrt JC, Aass AM, Koldsland OC. Treatment of peri-implant mucositis with a chitosan brush-A pilot randomized clinical trial. Int J Dent Hyg. (2019) 17:170-6. doi: 10.1111/idh.12381

24. Toma S, Brecx MC, Lasserre JF. Clinical evaluation of three surgical modalities in the treatment of peri-implantitis: a randomized controlled clinical trial. $J$ Clin Med. (2019) 8:966. doi: 10.3390/jcm8070966

25. Blasi A, Iorio-Siciliano V, Pacenza C, Pomingi F, Matarasso S, Rasperini G. Biofilm removal from implants supported restoration using different instruments: a 6-month comparative multicenter clinical study. Clin Oral Implants Res. (2016) 27:e68-73. doi: 10.1111/clr.12530

26. Pommer B, Haas R, Mailath-Pokorny G, Furhauser R, Watzek G, Busenlechner D, et al. Periimplantitis treatment: long-term comparison of laser decontamination and implantoplasty surgery. Implant Dent. (2016) 25:646-9. doi: 10.1097/ID.0000000000000461

27. Ellingsen JE. Thematic abstract review: implantoplasty: a valuable method for the management of peri-implantitis? Int J Oral Maxillofac Implants. (2017) 32:711-4. doi: 10.11607/jomi.2017.4.tar

28. Schwarz F, John G, Schmucker A, Sahm N, Becker J. Combined surgical therapy of advanced peri-implantitis evaluating two methods of surface decontamination: a 7-year follow-up observation. J Clin Periodontol. (2017) 44:337-42. doi: 10.1111/jcpe.12648

29. Schwarz F, Mihatovic I, Golubovic V, Becker J, Sager M. Immunohistochemical characteristics of regenerated bone after surgical therapy of advanced ligature-induced peri-implantitis defects. Clin Oral Investig. (2014) 18:1679-86. doi: 10.1007/s00784-013-1138-5

30. Stavropoulos A, Bertl K, Eren S, Gotfredsen K. Mechanical and biological complications after implantoplasty-A systematic review. Clin Oral Implants Res. (2019) 30:833-48. doi: 10.1111/clr.13499

31. Figuero E, Graziani F, Sanz I, Herrera D, Sanz M. Management of periimplant mucositis and peri-implantitis. Periodontol 2000. (2014) 66:25573. doi: $10.1111 /$ prd.12049

32. Shibli JA, Ferrari DS, Siroma RS, Figueiredo LC, Faveri M, Feres M. Microbiological and clinical effects of adjunctive systemic metronidazole and amoxicillin in the non-surgical treatment of peri-implantitis: 1 year follow-up. Braz Oral Res. (2019) 33 (Suppl. 1):e080. doi: 10.1590/1807-3107bor-2019.vol33.0080

33. Yoon SW, Kim MJ, Paeng KW, Yu KA, Lee CK, Song YW, et al. Efficacy of local minocycline agents in treating peri-implantitis: an 
experimental in vivo study in beagle dogs. Pharmaceutics. 12:1016. doi: 10.3390/pharmaceutics12111016

34. Khan A, Goyal A, Currell SD, Sharma D. Management of peri-implantitis lesions without the use of systemic antibiotics: a systematic review. Dent J. (2020) 8:106. doi: 10.3390/dj8030106

35. Yi Y, Koo KT, Schwarz F, Ben Amara H, Heo SJ. Association of prosthetic features and peri-implantitis: a cross-sectional study. J Clin Periodontol. (2020) 47:392-403. doi: 10.1111/jcpe.13251

36. Cohen J. Statistical Power Analysis for the Behavioral Sciences. 2nd ed. Hillsdale, NJ: Lawrence Erlbaum Associates (1988).

37. Kane G, Fang V, Simon S, Girdler J, Adeyinka O, Alhilou A. A survey exploring the experiences \& attitudes of dental implant clinicians in the management of peri-implantitis within the United Kingdom. Eur J Prosthodont Restor Dent. (2018) 26:46-52. doi: 10.1922/EJPRD_01761Kane07

38. Mattheos N, Collier S, Walmsley AD. Specialists' management decisions and attitudes towards mucositis and peri-implantitis. Br Dent J. (2012) 212:E1. doi: 10.1038/sj.bdj.2012.1

39. Papathanasiou E, Finkelman M, Hanley J, Parashis AO. Prevalence, etiology and treatment of peri-implant mucositis and peri-implantitis: a survey of periodontists in the United States. J Periodontol. (2016) 87:493501. doi: 10.1902/jop.2015.150476

40. Sanz M, Noguerol B, Sanz-Sanchez I, Hammerle CHF, Schliephake H, Renouard F, et al. European Association for osseointegration delphi study on the trends in implant dentistry in Europe for the year 2030. Clin Oral Implants Res. (2019) 30:476-86. doi: 10.1111/clr.13431

41. Abrahamsson KH, Wennstrom JL, Hallberg U. Patients' views on periodontal disease; attitudes to oral health and expectancy of periodontal treatment: a qualitative interview study. Oral Health Prev Dent. (2008) 6:209-16

42. Listl S, Fruhauf N, Dannewitz B, Weis C, Tu YK, Chang HJ, et al. Costeffectiveness of non-surgical peri-implantitis treatments. J Clin Periodontol. (2015) 42:470-7. doi: 10.1111/jcpe.12402

43. Schwendicke F, Tu YK, Stolpe M. Preventing and treating periimplantitis: a cost-effectiveness analysis. J Periodontol. (2015) 86:1020-9. doi: 10.1902/jop.2015.150071

44. Toma S, Lasserre J, Brecx MC, Nyssen-Behets C. In vitro evaluation of periimplantitis treatment modalities on Saos-2osteoblasts. Clin Oral Implants Res. (2016) 27:1085-92. doi: 10.1111/clr.12686

45. Herrera D, Serrano J, Roldan S, Sanz M. Is the oral cavity relevant in SARS-CoV-2 pandemic? Clin Oral Investig. (2020) 24:2925-30. doi: 10.1007/s00784-020-03413-2

46. Herrera D, Alonso B, León R, Roldán S, Sanz M. Antimicrobial therapy in periodontitis: the use of systemic antimicrobials against the subgingival biofilm. J Clin Periodontol. (2008) 35:45-66. doi: 10.1111/j.1600-051X.2008. 01260.x

47. Kapoor A, Malhotra R, Grover V, Grover D. Systemic antibiotic therapy in periodontics. Dent Res J (Isfahan). (2012) 9:50515. doi: 10.4103/1735-3327.104866

48. Aslam S. Effect of antibacterials on biofilms. Am J Infect Control. (2008) 36:S175 e9-11. doi: 10.1016/j.ajic.2008.10.002

49. Ciofu O, Rojo-Molinero E, Macia MD, Oliver A. Antibiotic treatment of biofilm infections. APMIS. (2017) 125:304-19. doi: 10.1111/apm. 12673

50. Brown MR, Collier PJ, Gilbert P. Influence of growth rate on susceptibility to antimicrobial agents: modification of the cell envelope and batch and continuous culture studies. Antimicrob Agents Chemother. (1990) 34:16238. doi: 10.1128/AAC.34.9.1623

51. La Fauci V, Alessi V. Antibiotic resistance: where are we going? Ann Ig. (2018) 30 (4 Suppl. 1):52-7. doi: 10.7416/ai.2018.2235

52. Geerts SO, Nys M, De MP, Charpentier J, Albert A, Legrand V, et al. Systemic release of endotoxins induced by gentle mastication: association with periodontitis severity. J Periodontol. (2002) 73:73-8. doi: 10.1902/jop.2002.73.1.73

53. Ohki T, Itabashi Y, Kohno T, Yoshizawa A, Nishikubo S, Watanabe S, et al. Detection of periodontal bacteria in thrombi of patients with acute myocardial infarction by polymerase chain reaction. Am Heart J. (2012) 163:164-7. doi: 10.1016/j.ahj.2011.10.012

54. Jepsen K, Jepsen S. Antibiotics/antimicrobials: systemic and local administration in the therapy of mild to moderately advanced periodontitis. Periodontol 2000. (2016) 71:82-112. doi: 10.1111/prd.12121

55. Rams TE, Degener JE, van Winkelhoff AJ. Antibiotic resistance in human peri-implantitis microbiota. Clin Oral Implants Res. (2014) 25:8290. doi: $10.1111 /$ clr.12160

56. Bolstad AI, Saetre MM, Aasgaard AS, Bunaes DF. Shift in antibiotic prescription at a University Dental Clinic in Norway 2013-2017. Eur J Oral Sci. (2020) 128:518-25. doi: 10.1111/eos.12741

57. Khan A, Sharma D. Management of peri-implant diseases: a survey of Australian periodontists. Dent J. (2020) 8:100. doi: 10.3390/dj8030100

58. van Winkelhoff AJ, Herrera D, Oteo A, Sanz M. Antimicrobial profiles of periodontal pathogens isolated from periodontitis patients in the Netherlands and Spain. J Clin Periodontol. (2005) 32:893-8. doi: 10.1111/j.1600-051X.2005.00782.x

59. Cassini A, Hogberg LD, Plachouras D, Quattrocchi A, Hoxha A, Simonsen GS, et al. Attributable deaths and disability-adjusted life-years caused by infections with antibiotic-resistant bacteria in the EU and the European Economic Area in 2015: a population-level modelling analysis. Lancet Infect Dis. (2019) 19:56-66. doi: 10.1016/S1473-3099(18)30605-4

60. Gomi K, Matsushima Y, Ujiie Y, Shirakawa S, Nagano T, Kanazashi M, et al. Full-mouth scaling and root planing combined with azithromycin to treat peri-implantitis. Aust Dent J. (2015) 60:503-10. doi: 10.1111/adj.12257

61. Renvert S, Quirynen M. Risk indicators for peri-implantitis. A narrative review. Clin Oral Implants Res. (2015) 26 (Suppl. 11):15-44. doi: 10.1111/clr.12636

62. Rosing CK, Fiorini T, Haas AN, Muniz F, Oppermann RV, Susin C. The impact of maintenance on peri-implant health. Braz Oral Res. (2019) 33 (Suppl. 1):e074. doi: 10.1590/1807-3107bor-2019.vol33.0074

63. Schwarz F, Derks J, Monje A, Wang HL. Peri-implantitis. J Periodontol. (2018) 89 (Suppl. 1):S267-S90. doi: 10.1002/JPER.16-0350

64. Heitz-Mayfield LJ, Heitz F, Lang NP. Implant disease risk assessment IDRAa tool for preventing peri-implant disease. Clin Oral Implants Res. (2020) 31:397-403. doi: 10.1111/clr.13585

65. Lang NP, Tonetti MS. Periodontal risk assessment (PRA) for patients in supportive periodontal therapy (SPT). Oral Health Prev Dent. (2003) 1:7-16.

66. Kalsi JS, Hemmings K. The influence of patients' decisions on treatment planning in restorative dentistry. Dent Update. (2013) 40:698-700, 2-4, 7-8, 10. doi: 10.12968/denu.2013.40.9.698

Conflict of Interest: The authors declare that the research was conducted in the absence of any commercial or financial relationships that could be construed as a potential conflict of interest.

The handling editor declared a shared affiliation with one of the authors ASt at time of review.

Publisher's Note: All claims expressed in this article are solely those of the authors and do not necessarily represent those of their affiliated organizations, or those of the publisher, the editors and the reviewers. Any product that may be evaluated in this article, or claim that may be made by its manufacturer, is not guaranteed or endorsed by the publisher.

Copyright (C) 2021 Hussain, Haugen, Aass, Sanz, Antonoglou, Bouchard, Bozic, Eickholz, Jepsen, Jepsen, Karaca, Kuru, Nemcovsky, Papapanou, Pilloni, Renvert, Roccuzzo, Sanz-Esporrin, Spahr, Stavropoulos, Verket, Vražić and Lyngstadaas. This is an open-access article distributed under the terms of the Creative Commons Attribution License (CC BY). The use, distribution or reproduction in other forums is permitted, provided the original author(s) and the copyright owner(s) are credited and that the original publication in this journal is cited, in accordance with accepted academic practice. No use, distribution or reproduction is permitted which does not comply with these terms. 